\title{
LA PSICOLOGÍA CLÍNICA: UNA REFLEXIÓN
}

\author{
Alfonso Blanco Picabia \\ Luis Rodriguez Franco \\ Universidad de Sevilla (España)
}

\section{RESUMEN}

En nuestro estudio, realizamos una reflexión acerca del estado actual de la Psicología Clínica, destacando tanto el desarrollo conceptual y empirico como el profesional que le ha caracterizado durante las últimas décadas. Analizamos algunos temas de interés sobre los que presentamos nuestras reflexiones, centrándonos en las estrategias de afrontamiento, los trastomos de la personalidad y el análisis de la eficacia terapéutica basadas en el meta-análisis

Palabras Clave: Psicologia Clínica, reflexiones, estrategias de afrontamiento, trastornos de la Personalidad, meta-análisis

\section{ABSTRACT}

In our study, we conducted a reflection on the current state of Clinical Psychology, highlighting both conceptual and empirical developments and also professional cues that have characterized the last few decades. We analyze some items of interest about where we presented our thoughts, focusing on coping strategies, personality disorders and analysis of therapeutic efficacy based on the meta-analysis method.

Keywords: Clinical Psychology, review, coping strategies, personality disorders, meta-analysis.

La correspondencia sobre este trabajo debe dirigirse a picabia@us.es / lurodri@ us.es. La responsabilidad de lo expuesto en el trabajo es exclusivamente de los autores. 


\section{INTRODUCCIÓN}

La Psicologia Clínica en España se encuentra en una buena situación vital y las expectativas no son menos esperanzadoras, al menos si la comparamos con la existente hace 30 años. Desde el punto de vista de la investigación las revistas científicas españolas sobre la materia han incrementado notoriamente el número de sus publicaciones, han aparecido algunas nuevas publicaciones muy relevantes y con buena esperanza de vida y otras han terminado consolidándose definitivamente en el panorama cientifico español e internacional; a nivel docente y formativo se vienen impartiendo enseñanzas especificas de grado y postgrado que han obtenido muy buena aceptación entre los discentes y los profesionales; y profesionalmente, se ha creado ¡Por fin! el título de Especialista en Psicología Clínica (aunque constituya un capítulo aún no cerrado)... Y es que sigue viva y en desarrollo, como desde sus inicios.

$Y$ decimos esto porque si acudimos a los primeros escritos publicados de los que tengamos constancia sobre revisiones de la Psicología Clinica, existen intereses y preocupaciones actuales coincidentes en algunos casos con las ya presentes hace más de ocho decenios. Sólo como ejemplo de la ya larga historia de la disciplina, Fearing y Franklin en 1925 de forma independiente disponen de la suficiente información como para poder efectuar ya entonces una revisión acerca de las aportaciones de la Psicologia Clínica al campo de la Psicologia, mientras que Woodworth en 1937 apuntaba los problemas y las expectativas acerca de la misma, aunque no todas sus predicciones más pesimistas se han visto cumplidas (Sechrest, 1992).

Cuanto menos, resulta destacable que los aspectos formativos sobre la formación en Psicología Clínica que hoy día están de plena actualidad sean precisamente los que interesaban en las publicaciones anteriores, como las de Doll (1939) entre muchas otras posteriores y especialmente en las décadas de los 40 en adelante.

$Y$ es precisamente esta inquietud por mejorar la que, a nuestro juicio, ha permitido el desarrollo de nuestra disciplina. Por lo que entendemos que se hace precisa una reflexión acerca de ciertos aspectos, que responden no tanto a la consideración de que se encuentre 
en una situación especialmente difícil, sino más bien al hecho de que la historia natural de cualquier materia necesita de momentos de reconsideración, de pararse a pensar.

Sólo a modo de reflexión de los autores y con este ánimo y propósito sugerimos algunos campos que estimamos pueden ser de interés para no ya "curar" a la Psicología Clínica (que no adolece de enfermedad relevante) sino para mejorar su "calidad de vida" en vista de que le auguramos muchos años de existencia fructífera.

Un punto de partida para estas reflexiones es la consideración de que no sólo son relevantes las publicaciones con elevado número de sujetos y amplia variedad de análisis multivariados. Otro tipo de análisis, como el de este número, son necesarios porque la forma de trabajar en Clínica también ha evolucionado y debe seguir cambiando a pesar del buen estado de salud. $Y$ exponemos una muestra.

Algunos recordaremos el software de Análisis Factorial realizado en los años 80 en los ordenadores Spectrum (Que debemos agradecer al profesor Seoane que en aquel momento nos lo hizo asequible). El proceso de análisis esencialmente consistía en mucho pensar y en tomar muchos cafés. Lo de pensar era inevitable después de conocer lo bien ( $y$ lento debido a las capacidades del procesador utilizado) que funcionaba el programa. Lo de los cafés era necesario para hacer llevadera la espera de las más o menos ocho horas que tardaba en terminar el proceso de cálculo. Con lo cual el investigador tenia que pensar (y más de lo que pensaba), lo que buscaba antes de iniciar una prueba para comprobar cómo o qué le aparecían en los resultados. Ese mismo proceso de cálculo (y en algunos casos, el razonamiento que le deberia guiar) se realiza hoy día en cualquier procesador en no más de un segundo.

La segunda muestra la recogemos de la experiencia de uno de los autores cuando intentó realizar una búsqueda bibliográfica en las bases de datos del Psychological Abstracts por la misma época. Le extrañó que para hacerla se tuviera que afinar tanto y buscar tantos términos cercanos o más amplios o más restringidos en el Thesaurus de esta empresa, hasta que descubrió que la búsqueda se realizaba, de hecho, a través de una conferencia transoceánica mediante un modem que funcionaba, con suerte, a $56 \mathrm{~Kb}$. Hoy dia tardamos lo mismo que para un análisis factorial. 
Entiéndase lo que sigue a modo de reflexión, a modo de opiniones. $Y$ sobre las opiniones y los gustos, ya se sabe, no hay manual empíricamente validado que indique cuál es la mejor. Ni tan siquiera es nuestra intención hacer un muestreo representativo.

\section{LAS ESTRATEGIAS DE AFRONTAMIENTO O ¿CÓMO ANALIZAMOS LA INVESTIGACIÓN?}

La relevancia de la estrategias de afrontamiento, entre muchos otros procesos psicológicos dentro del amplio espectro de situaciones que necesitan ser atendidas por la Psicología Clinica, radica básicamente en que los pacientes que usualmente atendemos han realizado un uso intensivo y prolongado de ellas con una nula o muy escasa eficacia, probablemente de forma insistente y reiterada, y en consecuencia, se perciben a si mismos y se sienten, como incapaces de solucionar su situación conflictiva. Y como consecuencia, buscan la ayuda del profesional.

Formuladas inicialmente por Lazarus y Folkman y revisadas posteriormente (Folkman, Lazarus, Dunkel-Schetter, DeLongis, y Gruen, 2000) han cambiado de formulación en aspectos relevantes que no siempre son recogidos adecuadamente en la investigación.

El análisis de estas "estrategias" es un proceso que se pone en marcha ante una situación "estresante" que requiere de esfuerzos de ajuste y adaptación por parte del sujeto. La primera observación proviene precisamente del tipo de esfuerzo que puede emplear la persona. De hecho, no tenemos conocimiento de publicaciones especificas que traten de aclarar o al menos de organizar, las distintas posibilidades de las formas de esfuerzo que puede desarrollar los humanos ante las situaciones que les resultan conflictivas. Más al contrario, conocemos de muchas publicaciones donde aparecen los muy variados tipos de esfuerzos que pueden ser evaluados a través de una gran diversidad de instrumentos especificos. Sin que exista una articulación o jerarquización clara de cuáles pueden ser realmente empleados.

Otrosi es que puedan ser adecuadamente clasificadas. Así, nos hallamos ante algunas que se describen como orientadas a la solución de problemas mientras que otras se encaminan más hacia la re- 
solución de las secuelas emocionales del mismo. Aunque este tipo de asignación produce casos en que unas y otras pueden ser confundidas o simplemente intercambiadas entre sí. En otros casos y simplemente como ejemplo, se trata de establecer una distinción entre las que consideramos "adaptativas" y las que llamamos "desadaptativas". Como si alguna estrategia en concreto, o agrupación de ellas, pudiese ser considerada como varita mágica que abriese la puerta de la solución de cualquier problema (véase, Suls y Fletcher, 1985, por ejemplo). Si ello fuese así, se nos antoja considerar que los esfuerzos que ha realizado la Psicología Clínica por diseñar complejos sistemas terapéuticos con objetivos variados deberían, en su caso, derivarse a identificar las Estrategias de Afrontamiento adecuadas para tipos de situaciones conflictivas concretas, tarea que se nos antoja poco fácil de realizar. Pero no por ello menos necesarias.

Con independencia de este tipo de clasificaciones, entendemos que una persona, al enfrentarse a una situación que considera problemática, pone en marcha toda su artillería psicológica personal más pesada. Lo que significa que en el proceso de afrontamiento empleará no una sino muchas estrategias de forma simultánea y no siempre con la constancia necesaria. Ello es fácilmente comprobable tan sólo analizando las medias que alcanzan los sujetos objeto de investigación en todas y cada una de las estrategias de afrontamiento que han sido evaluadas o simplemente observando los índices de correlación que existen entre ellas.

Sin embargo el análisis matemático que de ello se hace es bien distinto por cuanto que casi sistemáticamente las estrategias de afrontamiento se procesan estadisticamente de forma aislada e individual. $Y$ en consecuencia, de manera muy distinta al cómo las emplean las personas. No conocemos suficientes estudios, por ejemplo, que analicen, sin más, si el emplear muchas estrategias diferentes, con independencia de cuáles sean, sea más eficaz que utilizar un menor número de ellas para la solución de la problemática del paciente. $O$, valga el ejemplo, cuáles de las posibles combinaciones de las EA es más eficaz para el mismo propósito, habida cuenta de que son utilizadas por la persona de manera simultánea. En cualquier caso, en los análisis estadisticos usuales (y siempre hay excepciones) se analiza el efecto de aislado de estrategias concretas, más que el combinado y natural. 
Por otro lado, las EA (y su eficacia) no siempre son utilizadas ni de la misma manera ni con la misma profusión durante el desarrollo del problema que aqueja al paciente (Pelechano, 2005). En fases tempranas del problema son utilizadas algunas, con niveles de eficacia probablemente distintos, a las que producen en las fases finales del mismo. Pensemos, por ejemplo, en un paciente de cáncer con mal pronóstico, que requiere asesoramiento psicológico. Evidentemente, sin saber el estado, fase o momento concreto y objetivo en el que psicológica y orgánicamente se encuentra, es prácticamente imposible decidir las EA que serian más relevantes para mejorar su estado. $Y$ es que las EA cambian su uso y su eficacia aunque poca investigación se dedique a ello.

Tampoco las EA se desarrollan en el vacío. Queremos señalar que quien implementa las EA es la persona y que "algo" debe contribuir a su empleo o a su eficacia. Preguntas como ¿Es igualmente probable que cualquier persona utilice la misma EA ante la misma situación? $\mathrm{O} ¿$ Cualquier EA es igualmente eficaz para todas las personas ante una misma situación? han empezado a formularse sólo de forma relativamente reciente (Suls y Fletcher, 1985). Sería poco convincente considerar que extravertidos e introvertidos emplean el Apoyo Social como EA con la misma frecuencia y eficacia o de la misma manera, o que, como otro ejemplo, que pacientes con razonamientos emocionales habituales utilicen la negación de la misma forma que aquellos otros con mayor rigidez intelectiva.

Esto conlleva, evidentemente, una notable y notoria complicación en los diseños y análisis de investigación pero que en ningún caso seria mayor (ni tendría que ser) que la propia del ser humano.

\section{LOS TRASTORNOS DE LA PERSONALIDAD O Cómo SABEMOS LO QUE DIAGNOSTICAMOS}

No debe caber duda que uno de los intereses, a nivel mundial, de la Psicología Clínica son los Trastornos de la Personalidad y más, si cabe, de forma reciente. Si observamos 30 años atrás identificando publicaciones periódicas sobre este tema, hallaremos que del total de artículos publicados el $42,21 \%$ lo han sido en los últimos ocho años (desde el año 2000). Esto es así tanto en las bases del Psycinfo como en las propias del MedLine (40,05\% en este último caso). 
¿Qué explica este reciente interés? Algunos aspectos pueden ser señalados. Por una parte, el diagnóstico de cualquier Trastorno de la Personalidad (TP) en sí o en combinación con algún otro trastorno, complica el curso y la evolución del cuadro del paciente en comparación a si tan sólo aparece un trastorno del Eje I. Lo cual consecuentemente, ha hecho plantear una importante cantidad de esfuerzos en desarrollar estrategias específicas de intervención eficaces.

Por otro lado, un importante volumen de publicaciones se ha dedicado a la delimitación, aclaración y mejor definición de los TP. En este sentido, cabe recordar que en la clínica los TP aparecen de forma comórbida y casi de manera sistemática con otras condiciones psicopatológicas, sean del Eje I o del II (Carolyn y Drew, 2005, por ejemplo). Con ello, nos podemos plantear qué son especificamente, en la práctica, esos TP que casi tan sólo de forma teórica aparecen descritos con nitidez y de forma diferenciada entre si. $\mathrm{O}$ al menos eso es lo que podría parecer en un primer momento.

En este sentido, el DSM-IV recoge como definición que los TP son un "patrón permanente e inflexible de experiencia interna y de comportamiento que... es estable a lo largo del tiempo y comporta malestar o perjuicios para el sujeto" (pág. 645). Caben al menos dos reflexiones ante tal definición: por un lado cabria plantear cuáles son las posibilidades terapéuticas para un trastorno que intrínsecamente es definido como "permanente e inflexible" en relación a otros que pudieran describirse dentro de un marco de referencia de menor rigidez. Pensamos que si una "entidad diagnóstica" es descrita de manera similar a como se realizan los diagnósticos de patologías irreversibles, el resultado terapéutico será muy probablemente mucho más limitado que si se describe en otros términos. En palabras de menor rigor, quizás al realizar el diagnóstico de TP con los criterios actuales estaríamos sentenciando la cronicidad. También pudiera considerarse "flexibilizar" y suavizar los criterios diagnósticos para evitar el roce con la cronicidad o terminalidad de los TP, a costa de incrementar las ya elevadas tasas epidemiológicas existentes en el Eje II. Aunque con ello probablemente se hiciera posible realizar diagnósticos tempranos o iniciales de los trastornos y en consecuencia una mejora de la eficacia terapéutica. 
Pero la cuestión básica en este sentido no sería sólo la de determinar si los TP son permanentes e inflexibles (además de estables), sino también identificar si los criterios diagnósticos que los definen lo son y si lo son para todos (o la mayoria de) los pacientes diagnosticados (Shedler y Westen, 2004). El campo de investigación que se abriría de esta manera sería notoriamente complejo y muy cercano, además, al campo de interés de las productivas y no siempre consideradas publicaciones sobre consistencia de la conducta y de la personalidad, que comentaremos más adelante.

Por otro lado, existe un claro deseo e intencionalidad en el caso del sistema DSM por adscribirse a planteamiento clínicos más que teóricos (la "ateoricidad" del DSM). Una sencilla lectura del manual de este sistema de clasificación nos puede sacar de dudas. En la página 646 figura expresamente que "los rasgos de personalidad son patrones persistentes de percibir, relacionarse y pensar sobre el entorno y uno mismo que se ponen de manifiesto en una amplia gama de contextos sociales y personales... El clínico tiene que valorar la estabilidad de los rasgos de personalidad a lo largo del tiempo y en situaciones diferentes" (las cursivas son nuestras). Es una clara referencia al modelo de rasgos de personalidad y por si existiera alguna duda, transcribimos la definición que ya efectuaron Funder y Colvin en 1991: “... se ocupa de la identificación y estudio de los patrones de conducta que las personas manifiestan a lo largo del tiempo y entre distintas situaciones". Esta ¿no deseada? ¿no intencionada? adscripción del DSM al modelo de rasgos no supondría males mayores si se actuara en consecuencia con las reformulaciones de los rasgos acontecidas desde los años ochenta, iniciadas por clásica publicación de Mischel en 1968 y posteriormente aclaradas y desarrolladas por numerosos investigadores. En suma, desde las primeras apariciones de la descripción de los trastornos de la personalidad en el DSM (1952, en el DSM-1), pasando posteriormente por la consideración independiente del Eje II en la DSM-III y hasta nuestros dias con el DSM-IV-R), muy poco ha cambiado la definición de los trastornos de la personalidad, mientras que mucho lo han hecho los estudios sobre los rasgos de personalidad. Se ha pasado de la rigidez determinista del rasgo a planteamientos más flexibles e interaccionistas; de considerar al rasgo como estricta y permanentemente estable a 
plantear distintos niveles de consolidación en función de su naturaleza; pasamos de considerar la consistencia como propiedad inherente tan sólo de los rasgos, a poderla aplicar a personas y diferenciar sus niveles según situaciones; $y$ un largo etcétera de innovaciones que deberian pero no han tenido lugar durante las sucesivas modificaciones del DSM. Y se nota.

Una reflexión final por no ser prolijos ni exhaustivos. Esta deriva no ya del concepto de los TP sino de la manera en que éstos se diagnostican. El mismo manual indica que dentro de cada categoría diagnóstica del Eje II, "los items en cada grupo de criterios para cada uno de los trastornos de la personalidad especificos son enumerados en orden de importancia diagnóstica decreciente según los datos relevantes sobre eficiencia diagnóstica (cuando existen)" (pág. 646). En consecuencia, difícilmente podemos entender que, al final, en los requisitos diagnósticos se acuda exclusivamente al número (cantidad) de criterios cumplidos sin considerar para nada lo anteriormente mencionado. La más rápida consecuencia de ello supone considerar que para un mismo diagnóstico es posible encontrar tanto a pacientes que cubran los ítems diagnósticos más relevantes o prototipicos como a pacientes que presenten la sintomatología más "light" o menos relevante del mismo trastorno de la personalidad. La consecuencia clínica y de investigación de ello no es poco relevante, por cuanto que supone que en el mismo diagnóstico se verán incluidos pacientes muy representativos del trastorno y otros de menor nivel de prototipicidad. Lo que hace dudar de que las muestras de investigación utilizadas para determinar la eficacia terapéutica de determinadas estrategias de intervención sean mínimamente homogéneas. Únanse a lo anterior las comorbilidades entre los TP, tanto entre sí como con las categorías del Eje I, para comprender el interés actual en modificar los TP en las venideras revisiones del sistema DSM.

\section{LOS ESTUDIOS DE METAANÁLISIS O ¿DÓNDE SITUAMOS LAS DIFERENCIAS INDIVIDUALES?}

Estudios empíricamente validados y comprobados (Teóricamente "Ciencia basada en la evidencia") son procedimientos que cada vez suenan con mayor frecuencia en el campo de la Psicología y de 
la Medicina. No queremos entrar en detalles en el campo específico de sus valiosas aportaciones ni de sus limitaciones metodológicas (Bullock y Svyantek, 1985; Viechtbauer, 2007; Morris, 2008), sino de los planteamientos que apoyan el procedimiento y su relación con la intervención psicológica.

La Psicología Clínica se aplica, en líneas generales y como forma más habitual a sujetos individuales y en todo caso a un reducido número de ellos. Esto permite controlar las diferencias interindividuales y en su consecuencia, poder adaptar y reprogramar las estrategias de intervención a sus necesidades y problemas particulares; al menos eso es lo que habitualmente tendemos a realizar en las intervenciones clínicas generales.

El procedimiento de metaanálisis necesita de grupos de estudios y de sujetos que han sido sometidos a intervención. Y de esta manera son analizados los resultados. El contraste entre estos procedimientos facilita que en el último caso se pierda individualidad (lo que Lamiell (1997) denominó la Psicología de la Impersonalidad) y de esta manera una pérdida del manejo de la información que consideramos esencial analizar para el progreso de la Psicología Clinica. Por ende, la metodologia de la aplicación terapéutica es distinta a la empleada en la recogida de información sobre su eficacia en el meta-análisis. Aunque es posible realizar análisis de variables moduladoras de los efectos del tratamiento, este tipo de metodologías son mucho menos empleadas (Kemery, Mossholder y Dunlap, 1989; Shadish y Sweeney, 1991, Russell y Gilliland, 1995; Sherman, 1996, Roberts, Kuncel, Viechtbauer y Bogg, 2007; Cipriano y Corrado, 2007).

Por otro lado, en los estudios sobre eficacia del tratamiento suelen utilizarse medidas de control en las que se hace necesario, estadísticamente, encontrar diferencias significativas y/o relaciones entre las medidas de control inicial y las del postratamiento para conocer sus efectos. $Y$ no es extraño hallar, entre los resultados, cifras del porcentaje de sujetos que han encontrado mejoría, así como una explicación acerca de los procesos de muy diversa índole que los han podido justificar.

Lo que echamos de menos de forma habitual es el análisis del por qué, en ocasiones, no funciona como debería un determinado tratamiento en ciertas personas, cuando consideramos que, clínicamente, 
esta información es incluso más relevante que la anterior. Si se nos permite el burdo ejemplo, los que han mejorado han obtenido beneficios del tratamiento pero ¿cómo quedan quienes no mejoran?, ¿por qué no han mejorado?, ¿qué haría falta para que mejoraran? En este sentido, la atención que se dedica en la investigación a quienes no reciben la eficacia terapéutica es bien distinta a la de quienes reciben sus efectos positivos. $Y$ tampoco hay que olvidar que los tratamientos también pueden poseer efectos iatrogénicos especialmente cuando no son efectivos. Valga por ejemplo, el estado en que nos encontramos, a veces, a algunos pacientes con dolor crónico que finalmente son asistidos en Clínicas del Dolor, pero que han realizado un recorrido por largos pasillos, por eternos Servicios de Especialidades, con una extensa historia de fracasos y cuya primera tarea terapéutica casi es la de tratar de suprimir los perversos efectos del fracaso terapéutico anterior. Recordamos a ciertos maestros de profesión que nos indicaban al iniciar las prácticas clinicas que "al menos al paciente hay que dejarlo, cuanto menos, tal como llegó a la consulta".

$Y$ es que disponemos de muy escasa información al respecto porque dichos análisis no son habitualmente realizados y con ello no se recoge una información que estimamos que podria resultar de mucha utilidad para la mejora de las estrategias y técnicas de intervención. Y a ello, por qué no, habría que añadir los análisis sobre las "pérdidas muestrales" o abandonos terapéuticos que se producen (Wierzbicki y Gene, 1993).

$Y$ es claro que el metaanálisis, sin negar sus aportaciones, no permite obtener de forma satisfactoria esta información. No es nuestra posición la de tratar de plantear la ineficacia o utilidad de estos procedimientos, sino destacar que utilizados por sí solos no permiten recoger toda la información que se requiere: probablemente son necesarios. Pero no son suficientes.

Un último apunte en este sentido es que, habitualmente, no toda la población tiene la misma posibilidad de ser incluida con la misma probabilidad en los estudios metaanalíticos. De hecho, existen poblaciones que nunca han sido sometidas a ellos y probablemente no lo sean durante muchos años. Nos referimos a los propios terapeutas. En el campo de la Medicina, al menos en España, se ha trabajado con mucha mayor profusión que en el caso de los psicólogos. De 
hecho, hoy día existen no menos de 7 servicios asistenciales dedicados exclusivamente a la atención de médicos enfermos, pero ¿y con los psicólogos?

El informe de la American Psychological Association de 2006 comenta extensamente la naturaleza del problema, desde la variada definición del que denomina "impaired psychologist", que básicamente se refiere desde las dificultades para ejercer adecuadamente la profesión, hasta el estrés laboral que padecen los propios psicólogos, pasando por las propias y no pequeñas dificultades que favorece el ser un profesional que trata a pacientes y necesita reconocer la necesidad de ser él mismo tratado por sus mismos colegas profesionales. Por tanto, no es sólo un problema de estrés profesional, sino que aparece en un contexto más amplio que añade dificultades adicionales no siempre fácilmente solucionables (Sherman,1996).

Esta problemática ha sido planteada en el campo de la Medicina, especialmente a través del programa PAIME (Plan de Atención Integral al Médico Enfermo), aunque éste, en España lleva ya un mayor recorrido. Pero qué duda cabe que la integración de la Psicología Clínica en el sistema sanitario probablemente favorecerá una dinámica y contexto asistenciales que la harán más cercana a la problemática del médico. Afortunadamente, ya existen estudios al respecto (Benavides, Moreno-Jiménez, Garrosa \& González, 2002) fruto del esfuerzo de adaptación de la disciplina. Lo que hace pensar que la Psicología Clínica está bien viva, coleando, con mucho camino que recorrer y con unas enormes esperanzas ( $y$ también necesidad) de seguir haciéndolo durante muchos años

\section{BIBILIOGRAFÍA}

APA (2006). Advancing colleague assistance in professional psychology. www.apa.org/practice/ACCA_Monograph.pdf.

Benavides Pereira, A.M., Moreno-Jiménez, B., Garrosa Hernández, E. \& González Gutiérrez, J. L. (2002). La evaluación específica del sindrome de burnout en psicologos: el "inventario de burnout de psicologos". Clinica y salud, 13(3), 257-283.

Bullock R. J. \& Svyantek D. J. (1985). Analyzing Meta-Analysis: Potential Problems, an Unsuccessful Replication, and Evaluation Criteria. Journal of Applied Psychology, 70(1), 108-115. 
Carolyn, Z. C. \& Westen, D. (2005). Borderline Personality Disorder in Clinical Practice. American Journal of Psychiatry, 162, 867-875. Cipriani, A. \& Corrado, B. (2007). What is an individual patient data meta-analysis? Epidemiologia e Psichiatria Sociale, 16(3), 203-204.

Doll, E. A. (1939). Preparation for Clinical Psychology. Journal of Consulting and Clinical Psychology, 3, 137-140.

Fearing, F. S. (1925). Review of Clinical Psychology. Journal of Abnormal and Social Psychology, 20(3), 324-329.

Folkman, S., Lazarus, R. S., Dunkel-Schetter, C., DeLongis, A. \& Gruen, R. J. (2000). The dynamics of a stressful encounter. En Higgins, E.T. \& Kruglanski, A. W. (Eds), Motivational science: Social and personality perspectives (pp. 111-127). New York, NY: Psychology Press.

Franklin S. (1925). Review of Clinical Psychology. Journal of Abnormal and Social Psychology, 20(3), 324-329.

Funder, D. C. \& Colvin, C. R. (1991). Explorations in behavioral consistency: Properties of persons, situations, and behaviors. Journal of Personality and Social Psychology, 60, 773-794.

Kemery, E. R., Mossholder, K. W \& Dunlap, W. P. (1989). Meta-analysis and moderator variables: A cautionary note on transportability. Journal of Applied Psychology. 74(1), 168-170.

Lamiell, J. T. (1997). Psicologia de la Personalidad: un estudio epistemológico. Promolibro. Valencia.

Morris, S. B. (2008). Review of Methods of meta-analysis: Correcting error and bias in research findings. Organizational Research Methods, 11(1), 184-187.

Pelechano Barberá, V. (2005). Acerca de afrontamiento y enfermedad crónica: una reflexión ¿fundada? con alguna sugerencia. Revista de Psicopatología y Psicología Clínica, 10 (1), 3-14.

Roberts, B. W., Kuncel, N. R., Viechtbauer, W. \& Bogg, T. (2007). Metaanalysis in personality psychology: A primer. En Robins, R. W., Fraley, R. C. \& Krueger, R. F. (Eds), Handbook of research methods in personality psychology (pp. 652-672). New York, NY, Guilford Press.

Russell, C.J. \& Gilliland, S. W. (1995). Why Meta-Analysls Doesn't Tell Us What the Data Really Mean: Distinguishing between Moderator Effects and Moderator Processes Journal of Management, 21(4), 813-831. 
Shadish, W. R. \& Sweeney, R. B. (1991). Mediators and Moderators in Meta-Anarysis: There's a Reason We Don't Let Dodo Birds Tell Us Which Psychotherapies Should Have Prizes. Journal of Consulting and Clinical Psychology, 59(6), 883-893.

Sechrest, L. (1992). The Past Future of Clinical Psychology: A Reflection on Woodworth (1937). Journal of Consulting and Clinical Psychology. 60(1), 18-23.

Shedler, J. \& Westen, D. (2004). Refining Personality Disorder Diagnosis: Integrating Science and Practice. American Journal of Psychiatry, 161, 1350-1365.

Sherman, M. O. (1996). Distress and professional impairment due to mental health problems among psychotherapists. Clinical Psychology Review, 16(4), 299-315.

Suls, J. \& Fletcher, B. (1985). The relative efficacy of avoidant and nonavoidant coping strategies: A meta-analysis. Health Psychology. 4(3), 249-288.

Viechtbauer, W. (2007). Review of Publication bias in meta-analysis: Prevention, assessment and adjustments. Psychometrika, 72(2), 269-271.

Wierzbicki, M. \& Gene, P. (1993). A meta-analysis of psychotherapy dropout. Professional Psychology: Research and Practice, 24(2), 190-195.

Woodworth, R. S. (1937). The future of clinical psychology. Journal of Consulting Psychology, 1(1), 4-5. 